\title{
Periodic Solution of a Neutral Delay Leslie Predator-Prey Model and the Effect of Random Perturbation on the Smith Growth Model
}

\author{
Tongtong Li and Wencai Zhao \\ College of Mathematics and Systems Science, Shandong University of Science and Technology, Qingdao 266590, China \\ Correspondence should be addressed to Wencai Zhao; zhaowencai@sdust.edu.cn
}

Received 18 October 2019; Revised 20 February 2020; Accepted 19 March 2020; Published 14 April 2020

Guest Editor: George V. Popescu

Copyright (C) 2020 Tongtong Li and Wencai Zhao. This is an open access article distributed under the Creative Commons Attribution License, which permits unrestricted use, distribution, and reproduction in any medium, provided the original work is properly cited.

\begin{abstract}
This paper puts forward a class of ratio-dependent Leslie predator-prey models. Firstly, a neutral delay predator-prey model with ratio dependence and impulse control is established and the existence of positive periodic solutions is proved by the coincidence degree theory. Secondly, a stochastic disturbance Leslie model of Smith growth is obtained when the interference of white noise is taken into consideration and the impact of delay is ignored. Applying Itô's formula, we get the conditions of system persistence and extinction. Finally we verify the correctness of theoretical analysis with numerical simulations.
\end{abstract}

\section{Introduction}

In the population dynamic systems, the relationship between population growth rate and population density is complex and diverse. The Verhulst-Pearl logistic equation [1]

$$
\dot{x}(t)=r x(t)\left(1-\frac{x(t)}{K}\right),
$$

is widely used to describe population growth for its concise mathematical expression and clear biological meaning, where $x(t)$ denotes the population density at time $t, r$ is the intrinsic growth rate, and $K$ is the environmental capacity. However, in 1963, Smith [2] studied the population dynamics of Daphnia magna and found that the growth of Daphnia magna population in the laboratory did not satisfy the above growth model, and he proposed the following growth equation:

$$
\dot{x}(t)=r x(t)\left(1-\frac{x(t)}{K}-\frac{\dot{x}(t)}{c K}\right),
$$

which is usually called the Smith growth equation, where $c$ is a positive constant. Then, in 1991, Kuang [3] considered a neutral predator-prey model with time delays and the Smith growth as follows:

$$
\left\{\begin{array}{l}
\dot{x}(t)=r x(t)\left(1-\frac{x(t-\tau)+\rho \dot{x}(t-\tau)}{K}\right)-y(t) p(x(t)), \\
\dot{y}(t)=y(t)(-\alpha+\beta p(x(t-\sigma))),
\end{array}\right.
$$

where $\tau$ and $\sigma$ are the recovery time delay of the prey population and the digestion time delay of the predator population, respectively, $\alpha$ is the death rate of the predator, and $\beta$ is the conversion factor of prey into the predator. $(\rho \dot{x}(t-\tau) / K)$ is the neutral delay term. $p(x)$ indicates the functional response of predator $y$ to prey $x$ and depends only on the density of the prey population. However, when the predators have to search for food, there will be competition among individual predators for limited prey. The amount of food the predator obtains depends not only on the density of the prey population but also on the density of the predator itself, which is influenced by the relative ratio of the number of predators and prey populations, reflecting the ratio-dependent characteristics. Leslie proposed the 
following Leslie model $[4,5]$ in studying the relationship between the predator and prey:

$$
\left\{\begin{array}{l}
\dot{x}(t)=x(t)\left(r_{1}-a_{1} x(t)-c_{1} y(t)\right), \\
\dot{y}(t)=y(t)\left(r_{2}-a_{2} \frac{y(t)}{x(t)}\right),
\end{array}\right.
$$

where $x(t)$ and $y(t)$ stand for prey and predator densities at time $t$, respectively. $r_{1}$ and $r_{2}$ are the intrinsic growth rates of the prey and predator, $a_{1}$ denotes the density-dependent coefficient of the prey, the parameter $c_{1}$ is the capturing rate, and $a_{2}$ is the conversion factor of prey into predators. Motivated by the works [3-5], we propose a ratio-dependent neutral delay Leslie predator-prey model as follows:

$$
\left\{\begin{array}{l}
\dot{x}(t)=r x(t)\left(1-\frac{x\left(t-\tau_{1}\right)}{K}-\varepsilon \dot{x}\left(t-\tau_{1}\right)\right)-\frac{e x(t) y(t)}{n y(t)+x(t)}, \\
\dot{y}(t)=a y(t)\left(1-b \frac{y\left(t-\tau_{2}\right)}{x\left(t-\tau_{2}\right)}\right)
\end{array}\right.
$$

where all of the parameters are positive and have the biological meanings listed in Table 1.

The first equation of the system states that the growth rate of prey depends not only on its own density but also on the neutral term. If there is no predator, the prey population will grow in Smith mode. The Smith model mainly describes the growth rates of the population under the condition of limited food. The relative growth rate of population size $(\dot{x}(t) / x(t))$ at time $t$ is proportional to the amount of food left before the time $\tau_{1}$. The food consumed by the population is mainly used for two purposes: the food needed to maintain the organism's own survival $\left(x\left(t-\tau_{1}\right) / K\right)$ and the food needed for population reproduction $\varepsilon \dot{x}\left(t-\tau_{1}\right)$. The denominator of functional response function $p=(e x /(n y+$ $x)$ ) contains $y$, which reflects the interaction among individuals within the predator population. The second equation implies that the predator population grow in Leslie mode
TABLE 1: Biological meanings of parameters for model (5).

\begin{tabular}{lc}
\hline Parameters & Description \\
\hline$\tau_{1}, \tau_{2}$ & The discrete delays \\
$r, a$ & The intrinsic growth rates of prey and predator \\
$K$ & The environmental capacity of the prey \\
$\varepsilon$ & The neutral coefficient of the prey \\
$e$ & The conversion rate \\
$n$ & The capture saturation rate \\
$b$ & The conversion factor of prey into predator \\
\hline
\end{tabular}

and the environmental capacity is proportional to prey population density.

Developing and utilizing biological populations in a proper way is an important issue facing humanity, which can make biological resources work for human beings and ensure the sustainability of ecosystems [6-12]. People's harvesting behavior is often not continuous, which may be once or several times at a certain time. For example, in fishery production, fishermen harvest fishes in few months and in agricultural production farmers spray pesticides at regular intervals to harvest (kill) pests. This harvesting method makes the population quantity or density be changed drastically in a short period of time, so it is more suitable to describe with impulsive differential equations. In the past 20 years, impulsive differential equations have been widely used in various biological models, for example, Zhao and Tang [13], Liu et al. [14], Li et al. [15], and Zhang et al. [16] introduced impulsive effects into the ordinary differential equation model. Li and Meng [17] and Qi et al. [18] established a class of impulsive stochastic differential equation models based on ordinary differential equations. $\mathrm{Du}$ and Feng [19] and Chen and $\mathrm{Du}$ [20] cooperated the impulsive control to the neutral predator-prey model, by using the coincidence degree theory, and they obtained the conditions for the existence of periodic solution. Then, based on $[19,20]$ and assuming that some parameters in system (5) are changed periodically and the population is pulse controlled, we will obtain the following neutral delay system with pulse effects:

$$
\left\{\begin{array}{l}
\dot{x}(t)=x(t)\left(r(t)-\frac{r(t) x\left(t-\tau_{1}(t)\right)}{K(t)}-\rho(t) \dot{x}\left(t-\tau_{1}(t)\right)-\frac{e(t) y(t)}{n y(t)+x(t)}\right), \\
\dot{y}(t)=a(t) y(t)\left(1-b(t) \frac{y\left(t-\tau_{2}(t)\right)}{x\left(t-\tau_{2}(t)\right)}\right) \\
\left.\begin{array}{l}
\Delta x\left(t_{k}\right)=-\theta_{1 k} x\left(t_{k}\right), \\
\Delta y\left(t_{k}\right)=-\theta_{2 k} y\left(t_{k}\right),
\end{array}\right\} t=t_{k}, k=1,2, \ldots,
\end{array}\right\}
$$


with initial conditions

$$
\begin{aligned}
& x(t)=p(t), p \in C^{1}([-\tau, 0],[0,+\infty)), p(0)>0, \\
& y(t)=q(t), q \in C^{1}([-\tau, 0],[0,+\infty)), q(0)>0,
\end{aligned}
$$

where $\Delta x\left(t_{k}\right)=x\left(t_{k}^{+}\right)-x\left(t_{k}\right), \Delta y\left(t_{k}\right)=y\left(t_{k}^{+}\right)-y\left(t_{k}\right), t_{k}$ is the pulse harvesting time, and $\theta_{i k}(i=1,2)$ is the pulse harvesting ratio. This implies that the harvest yield is in proportion to the biomass at that time. $r(t), K(t)$, $\tau_{1}(t), \tau_{2}(t), e(t), a(t), b(t)$, and $\rho(t)$ are continuous nonnegative T-periodic functions. $n$ is positive constant and $\tau:=\max _{t \in[0, T]}\left\{\tau_{1}(t), \tau_{2}(t)\right\}$.

On the contrary, biological populations multiply and thrive in nature, which will inevitably be affected by various environmental noise, so it is important to consider the effects of random disturbances on population dynamics
[21-29]. In [30], the asymptotic stability of a stochastic May mutualism system was studied. In [31], Markov semigroup was used to study a stochastic ecological model of plants with infectious diseases. Authors in [32-34] focused on the long-term dynamic behavior of ecosystems affected by environmental noise. In particular, conditions for the existence of stationary distribution were obtained. Zhao et al. [35] presented an algal population growth model, where the authors not only gave the threshold conditions of permanence and extinction but also discussed the influence of environmental noise on the periodic blooms. Ignoring the influence of time delays and considering the disturbance of white noise, we construct the following stochastic Leslie predator-prey model with Smith growth:

$$
\left\{\begin{array}{l}
\mathrm{d} x(t)=\left[\frac{r x(t)}{1+\rho x(t)}\left(1-\frac{x(t)}{K}\right)-\frac{e x(t) y(t)}{(n y(t)+x(t))(1+\rho x(t))}\right] \mathrm{d} t+\sigma_{1} x(t) d B_{1}(t) \\
\mathrm{d} y(t)=\left[a y(t)\left(1-\frac{b y(t)}{x(t)}\right)\right] \mathrm{d} t+\sigma_{2} y(t) d B_{2}(t)
\end{array}\right.
$$

where $B_{1}(t)$ and $B_{2}(t)$ are independent standard Brownian motions. $\sigma_{1}^{2}$ and $\sigma_{2}^{2}$ denote the intensity of white noise. $r, K, \rho, n, a, b, e, \sigma_{1}$, and $\sigma_{2}$ are all positive and the biological significances are the same as that in model (5).

To study the existence of periodic solutions for ordinary differential equations and delay differential equations, Gaines and Mawhin's continuation theorem based on coincidence degree theory [36] is an important tool. However, it is seldom applied in the study of the existence of positive periodic solutions of neutral delay system with pulse effects. It is generally considered difficult to prove that the nonlinear operator $H$ is compact on set $\bar{\Omega}$ and the solutions of the equation have prior bound. In this work, by using analytical methods such as the mean value theorem of integral and inequality techniques, we obtain the sufficient conditions for the existence of positive periodic solutions of system (6). In addition, considering the influence of environmental disturbance on the population, we include the white noise into the Leslie predator-prey system, establish a new stochastic Smith growth model (8), and discuss the conditions for the persistence and extinction of the system.

\section{Preliminaries}

First, we convert the impulse system (6) into a nonimpulsive form. In system (6), we assume that

$\left[I_{1}\right] 0<t_{1}<t_{2}<\cdots<t_{k}<\cdots$ are fixed points and $\lim _{k \rightarrow \infty} t_{k}=+\infty$

$\left[I_{2}\right]\left\{\theta_{i k}\right\}$ are real sequences such that $\theta_{i k}<1$ and $\prod_{0<t_{k}<t}\left(1-\theta_{i k}\right)(i=1,2)$ are $\mathrm{T}$-periodic functions

Assuming $\left[I_{1}\right]$ and $\left[I_{2}\right]$, we convert system (6) to the following system:

$$
\left\{\begin{array}{l}
\dot{D}_{1}(t)=D_{1}(t)\left[r(t)-A(t) D_{1}\left(t-\tau_{1}(t)\right)-\eta(t) \dot{D}_{1}\left(t-\tau_{1}(t)\right)-\frac{E(t) D_{2}(t)}{N(t) D_{2}(t)+\theta_{1}(t) D_{1}(t)}\right], \\
\dot{D}_{2}(t)=D_{2}(t)\left[a(t)-\frac{B(t) D_{2}\left(\left(t-\tau_{2}(t)\right)\right.}{\theta_{2}(t) D_{1}\left(t-\tau_{2}(t)\right)}\right],
\end{array}\right.
$$

with initial conditions

$$
\begin{array}{ll}
D_{1}(t)=p(t), & p \in C^{1}([-\tau, 0],[0,+\infty)), p(0)>0, \\
D_{2}(t)=q(t), & q \in C^{1}([-\tau, 0],[0,+\infty)), q(0)>0,
\end{array}
$$


where

$$
\begin{aligned}
& D_{1}(t)=\prod_{0<t_{k}<t}\left(1-\theta_{1 k}\right)^{-1} x(t), \\
& D_{2}(t)=\prod_{0<t_{k}<t}\left(1-\theta_{2 k}\right)^{-1} y(t), \\
& A(t)=\frac{r(t)}{K(t)} \prod_{0<t_{k}<t-\tau_{1}(t)}\left(1-\theta_{1 k}\right), \\
& \eta(t)=\rho(t) \prod_{0<t_{k}<t-\tau_{1}(t)}\left(1-\theta_{1 k}\right), \\
& E(t)=e(t) \prod_{0<t_{k}<t}\left(1-\theta_{2 k}\right), \\
& N(t)=n \prod_{0<t_{k}<t}\left(1-\theta_{2 k}\right), \\
& \theta_{1}(t)=\prod_{0<t_{k}<t}\left(1-\theta_{1 k}\right),
\end{aligned}
$$

$$
\begin{aligned}
\theta_{2}(t) & =\prod_{0<t_{k}<t-\tau_{2}(t)}\left(1-\theta_{1 k}\right), \\
B(t) & =a(t) b(t) \prod_{0<t_{k}<t-\tau_{2}(t)}\left(1-\theta_{2 k}\right) .
\end{aligned}
$$

Lemma 1. Suppose that $\left[I_{1}\right]$ and $\left[I_{2}\right]$ hold, then

(1) If $\left(D_{1}(t), D_{2}(t)\right)^{T}$ is a solution of (9), then $(x(t)$, $y(t))^{T}$ is a solution of (6)

(2) If $(x(t), y(t))^{T}$ is a solution of (6), then $\left(D_{1}(t)\right.$, $\left.D_{2}(t)\right)^{T}$ is a solution of (9)

Proof. (1) Suppose $\left(D_{1}(t), D_{2}(t)\right)^{T}$ is a solution of system (9). Next, we prove that $x(t)=\prod_{0<t_{k}<t}\left(1-\theta_{1 k}\right) D_{1}(t)$, $y(t)=\prod_{0<t_{k}<t}\left(1-\theta_{2 k}\right) D_{2}(t)$ is the solution of system (6). When $t \neq t_{k}$, we obtain

$$
\begin{aligned}
x^{\prime}(t) & -x(t)\left(r(t)-\frac{r(t) x\left(t-\tau_{1}(t)\right)}{K(t)}-\rho(t) x^{\prime}\left(t-\tau_{1}(t)\right)-\frac{e(t) y(t)}{n y(t)+x(t)}\right) \\
= & \prod_{0<t_{k}<t}\left(1-\theta_{1 k}\right) D_{1}^{\prime}(t)-\prod_{0<t_{k}<t}\left(1-\theta_{1 k}\right) D_{1}(t)\left(r(t)-\frac{r(t) \prod_{0<t_{k}<t-\tau_{1}(t)}\left(1-\theta_{1 k}\right) D_{1}\left(t-\tau_{1}(t)\right)}{K(t)}\right. \\
& \left.-\rho(t) \prod_{0<t_{k}<t-\tau_{1}(t)}\left(1-\theta_{1 k}\right) D_{1}^{\prime}\left(t-\tau_{1}(t)\right)-\frac{e(t) \prod_{0<t_{k}<t}\left(1-\theta_{2 k}\right) D_{2}(t)}{n \prod_{0<t_{k}<t}\left(1-\theta_{2 k}\right) D_{2}(t)+\prod_{0<t_{k}<t}\left(1-\theta_{1 k}\right) D_{1}(t)}\right) \\
= & \prod_{0<t_{k}<t}\left(1-\theta_{1 k}\right)\left[D_{1}^{\prime}(t)-D_{1}(t)\left(r(t)-A(t) D_{1}(t-\tau(t))-\eta(t) D_{1}^{\prime}\left(t-\tau_{1}(t)\right)-\frac{E(t) D_{2}(t)}{N(t) D_{2}(t)+\theta_{1}(t) D_{1}(t)}\right)\right] \\
= & 0,
\end{aligned}
$$$$
y^{\prime}(t)-y(t)\left(a(t)-\frac{a(t) b(t) y\left(t-\tau_{2}(t)\right)}{x\left(t-\tau_{2}(t)\right)}\right)=\prod_{0<t_{k}<t}\left(1-\theta_{2 k}\right)\left[D_{2}^{\prime}(t)-D_{2}(t)\left(a(t)-\frac{B(t) D_{2}\left(t-\tau_{2}(t)\right)}{\theta_{2}(t) D_{1}\left(t-\tau_{2}(t)\right)}\right)\right]
$$$$
=0 \text {. }
$$

When $t=t_{k}$, one has

$$
\begin{aligned}
x\left(t_{k}^{+}\right)-x\left(t_{k}\right) & =\prod_{0<t_{j} \leq t_{k}}\left(1-\theta_{1 j}\right) D_{1}\left(t_{k}\right)-\prod_{0<t_{j}<t_{k}}\left(1-\theta_{1 j}\right) D_{1}\left(t_{k}\right) \\
& =\prod_{0<t_{j}<t_{k}}\left(1-\theta_{1 j}\right) D_{1}\left(t_{k}\right)\left(-\theta_{1 k}\right) \\
& =-\theta_{1 k} x\left(t_{k}\right),
\end{aligned}
$$


Therefore, $(x(t), y(t))^{T}$ is the solution of system (6).

(2) Similar to the proof of case (1), we could show that if $(x(t), y(t))^{T}$ is a solution of (6), then $\left(D_{1}(t), D_{2}(t)\right)^{T}$ is the solution of system (9). The proof of Lemma 1 is completed.

For convenience, throughout this paper we will use the symbols:

$$
\begin{aligned}
& \bar{v}=\frac{1}{T} \int_{0}^{T} v(t) \mathrm{d} t, \\
& \widehat{v}=\min _{t \in[0, T]} v(t), \\
& \widehat{v}=\max _{t \in[0, T]} v(t),
\end{aligned}
$$

where $v=v(t)$ is a periodic continuous function with period $T$.

\section{Existence of Positive Periodic Solution of System (6)}

Before discussing the existence of periodic solution of system (6), we introduce the coincidence degree theory firstly.

Let $U$ and $V$ be two Banach spaces, $G$ : Dom $G \subset U \longrightarrow V$ is a linear mapping and $H: U \longrightarrow V$ is a continuous mapping. If $\operatorname{dim} \operatorname{Ker} G=\operatorname{codim} \operatorname{Im} G<+\infty$ and $\operatorname{Im} G \subset V$ is closed, then we call the operator $G$ is a Fredholm operator with index zero. If $G$ is a Fredholm operator with index zero and there exist continuous projections $P: U \longrightarrow U$ and Q: $V \longrightarrow V$ such that $\operatorname{Im} P=\operatorname{Ker} G$ and $\operatorname{Im} G=$ $\operatorname{kerQ}=\operatorname{Im}(I-Q)$; then, $\left.G\right|_{\text {DomG } \cap \operatorname{Ker} P}:(I-P) U \longrightarrow \operatorname{ImG}$ has an inverse function, and we set it as $K_{p}$. Assume that $\Omega \subset U$ is any open set, if $Q H(\bar{\Omega})$ is bounded and $K_{p}(I-$ Q) $H: \bar{\Omega} \longrightarrow U$ is relative compact, and then we say $H$ is $G$-compact on $\bar{\Omega}$.

Lemma 2 (see [36]). Let G:Dom $G \subset U \longrightarrow V$ be a Fredholm mapping with index zero and $H$ is a G-compact on $\bar{\Omega}$. Furthermore, assume that

(i) $G x \neq \lambda H x$, where $\lambda \in(0,1), x \in \partial \Omega \cap$ DomG

(ii) $Q H x \neq 0$, for each $x \in \partial \Omega \cap \operatorname{ker} G$ (iii) $\operatorname{deg}\{J Q H, \Omega \cap \operatorname{Ker} G, 0\} \neq 0$, where $J: \operatorname{ImQ} \longrightarrow$ $\operatorname{Ker} G$ is an isomorphism

Then, the operator equation $G x=H x$ has at least one solution in DomG $\cap \bar{\Omega}$.

Lemma 3 (see [37]). If $\tau \in C^{1}(R, R)$ with $\tau(t+T) \equiv \tau(t)$ and $\tau^{\prime}(t)<1$ for $t \in[0, T]$, then function $\beta(t)=t-\tau(t)$ has a unique inverse $\beta^{-1}(t)$ satisfying $\beta^{-1} \in C^{1}(R, R)$ with $\beta^{-1}(s+T) \equiv \beta^{-1}(s)+T$ for $s \in[0, T]$. result.

We are now in a position to state and prove our main

Theorem 1. Assume that the following conditions hold:

$$
\begin{aligned}
& {\left[H_{1}\right] \tau_{1}^{\prime}(t)<1, \tau_{2}^{\prime}(t)<1 \text { for any } t \in R} \\
& {\left[H_{2}\right] \mu^{\prime}(t)<A(t) \text { for any } t \in[0, T] \text {, where }} \\
& \mu(t)=\left(\eta(t) /\left(1-\tau_{1}^{\prime}(t)\right)\right) \\
& {\left[H_{3}\right] \bar{\eta} e^{W}<1, \bar{r}>\bar{M} \text {, where } W \text { and } M \text { are defined in the }} \\
& \text { proof } \\
& {\left[H_{4}\right] \text { The algebraic equations }} \\
& \qquad \bar{r}-\bar{A} x-\frac{1}{T} \int_{0}^{T} \frac{E(t) y}{N(t) y+\theta_{1}(t) x} \mathrm{~d} t=0, \\
& \qquad \bar{a}-\overline{\left(\frac{B}{\theta_{2}}\right)} \frac{y}{x}=0,
\end{aligned}
$$

have finite solutions. Then, system (6) has at least one Tperiodic solution.

In the following proof, we first transform (9) into equivalent system. Then, we construct the mappings and open set $\Omega$ on Banach space and prove that the mappings satisfy Lemma 2 on $\bar{\Omega}$. Therefore, we obtain Theorem 1 .

Proof

(1) Transform (9) into an equivalent system.

Let $D_{1}(t)=e^{w(t)}$ and $D_{2}(t)=e^{v(t)}$. Then, we can translate system (9) into

$$
\left\{\begin{array}{l}
w^{\prime}(t)=r(t)-A(t) e^{w\left(t-\tau_{1}(t)\right)}-\eta(t) w^{\prime}\left(t-\tau_{1}(t)\right) e^{w\left(t-\tau_{1}(t)\right)}-\frac{E(t) e^{v(t)}}{N(t) e^{v(t)}+\theta_{1}(t) e^{w(t)}}, \\
v^{\prime}(t)=a(t)-\frac{B(t) e^{v\left(t-\tau_{2}(t)\right)}}{\theta_{2}(t) e^{w\left(t-\tau_{2}(t)\right)}}
\end{array}\right.
$$

where all coefficient functions are defined in system (9). It is easy to see that if system (16) has one T-periodic solution $\left(w^{*}(t), v^{*}(t)\right)^{T}$, then $\left(D_{1}^{*}(t), D_{2}^{*}(t)\right)^{T}=\left(\exp \left\{w^{*}(t)\right\}\right.$, $\left.\exp \left\{v^{*}(t)\right\}\right)^{T}$ is a positive T-periodic solution of system (9). So, system (6) has a T-periodic solution $\left(x^{*}(t), y^{*}(t)\right)^{T}$ $=\left(\prod_{0<t_{k}<t}\left(1-\theta_{1 k}\right) D_{1}^{*}(t), \prod_{0<t_{k}<t}\left(1-\theta_{2 k}\right) D_{2}^{*}(t)\right)^{T}$. Therefore, we just have to prove system (16)s has one T-periodic solution.
(2) Construct Banach space and define mappings.

Take $U=V=\left\{u(t)=(w(t), v(t))^{T} \in C^{1}\left(R, R^{2}\right): u(t+\right.$ $T)=u(t)\}$ and denote $|u|_{\infty}=\max _{t \in[0, T]}(|w(t)|+|v(t)|)$, $\|u\|=|u|_{\infty}+\left|u^{\prime}\right|_{\infty}$. Then, $U$ and $V$ are both Banach spaces with the norm $\|\cdot\|$ and $|\cdot|_{\infty}$, respectively. Define operators $G, P, Q$, and $H$ in the following form, respectively, $G: U \longrightarrow V, G u(t)=\left(w^{\prime}(t), v^{\prime}(t)\right)^{T} ; P(u)=\bar{u}$, for $u \in U$; $Q(u)=\bar{u}$, for $u \in V$; and 


$$
H u(t)=\left(\begin{array}{c}
r(t)-A(t) e^{w\left(t-\tau_{1}(t)\right)}-\eta(t) w^{\prime}\left(t-\tau_{1}(t)\right) e^{w\left(t-\tau_{1}(t)\right)}-\frac{E(t) e^{v(t)}}{N(t) e^{v(t)}+\theta_{1}(t) e^{w(t)}} \\
a(t)-\frac{B(t) e^{v\left(t-\tau_{2}(t)\right)}}{\theta_{2}(t) e^{w\left(t-\tau_{2}(t)\right)}}
\end{array}\right) .
$$

Then, $\operatorname{Ker} G=R^{2}, \quad \operatorname{Im} G=\left\{u \in V: \int_{0}^{T} u(t) \mathrm{d} t=0\right\} \quad$ is closed in $V$, dimKerG $=\operatorname{codimImG} . P$ and $Q$ are continuous projectors such that $\operatorname{Im} P=\operatorname{Ker} G, \operatorname{Ker} Q=\operatorname{Im} G=\operatorname{Im}(I-Q)$. Therefore, the Fredholm mapping $G$ has a unique inverse. The generalized inverse(to $G) K_{p}: \operatorname{Im} G \longrightarrow \operatorname{Ker} P \cap \operatorname{Dom} G$ is given by the following form:

$$
K_{p}(u)=\int_{0}^{t} u(s) \mathrm{d} s-\frac{1}{T} \int_{0}^{T} \int_{0}^{t} u(s) \mathrm{d} s \mathrm{~d} t .
$$

Then, $Q H: U \longrightarrow V$ and $K_{p}(I-Q) H: U \longrightarrow U$ read

$$
\mathrm{QHu}(t)=\left(\begin{array}{c}
\frac{1}{T} \int_{0}^{T}\left(r(t)-A(t) e^{w\left(t-\tau_{1}(t)\right)}-\eta(t) w^{\prime}\left(t-\tau_{1}(t)\right) e^{w\left(t-\tau_{1}(t)\right)}-\frac{E(t) e^{v(t)}}{N(t) e^{v(t)}+\theta_{1}(t) e^{w(t)}}\right) \mathrm{d} t \\
\frac{1}{T} \int_{0}^{T}\left(a(t)-\frac{B(t) e^{v\left(t-\tau_{2}(t)\right)}}{\theta_{2}(t) e^{w\left(t-\tau_{2}(t)\right)}}\right) \mathrm{d} t
\end{array}\right),
$$

$K_{p}(I-Q) H u(t)=A-B-C$,

where

$$
\begin{aligned}
& A=\left(\begin{array}{c}
\int_{0}^{t}\left(r(s)-A(s) e^{w\left(s-\tau_{1}(s)\right)}-\eta(s) w^{\prime}\left(s-\tau_{1}(s)\right) e^{\left(w s-\tau_{1}(s)\right)}-\frac{E(s) e^{v(s)}}{N(s) e^{v(s)}+\theta_{1}(t) e^{w(s)}}\right) \mathrm{d} s \\
\int_{0}^{t}\left(a(s)-\frac{B(s) e^{v\left(s-\tau_{2}(s)\right)}}{\theta_{2}(t) e^{w\left(s-\tau_{2}(s)\right)}}\right) \mathrm{d} s
\end{array}\right), \\
& B=\left(\begin{array}{c}
\frac{1}{T} \int_{0}^{T} \int_{0}^{t}\left(r(s)-A(s) e^{w\left(s-\tau_{1}(s)\right)}-\eta(s) w\left(s-\tau_{1}(s)\right) e^{w\left(s-\tau_{1}(s)\right)}-\frac{E(s) e^{v(s)}}{N(s) e^{v(s)}+\theta_{1}(t) e^{w(s)}}\right) \mathrm{d} s \mathrm{~d} t \\
\frac{1}{T} \int_{0}^{T} \int_{0}^{t}\left(a(s)-\frac{B(s) e^{v\left(s-\tau_{2}(s)\right)}}{\theta_{2}(t) e^{w\left(s-\tau_{2}(s)\right)}}\right) \mathrm{d} s \mathrm{~d} t
\end{array}\right), \\
& C=\left(\begin{array}{c}
\left(\frac{t}{T}-\frac{1}{2}\right) \int_{0}^{T}\left(r(s)-A(s) e^{w\left(s-\tau_{1}(s)\right)}-\eta(s) w^{\prime}\left(s-\tau_{1}(s)\right) e^{w\left(s-\tau_{1}(s)\right)}-\frac{E(s) e^{v(s)}}{N(s) e^{v(s)}+\theta_{1}(t) e^{w(s)}}\right) \mathrm{d} s \\
\left(\frac{t}{T}-\frac{1}{2}\right) \int_{0}^{T}\left(a(s)-\frac{B(s) e^{v\left(s-\tau_{2}(s)\right)}}{\theta_{2}(t) e^{w\left(s-\tau_{2}(s)\right)}}\right) \mathrm{d} s
\end{array}\right) .
\end{aligned}
$$

Distinctly, it is easy to know that $\mathrm{QH}$ and $K_{p}(I-Q) H$ are both continuous by the Lebesgue theorem. And by using Arzela-Ascoli theorem, we know that $K_{p}(I-Q) H(\bar{\Omega})$ is compact for any open bounded set $\Omega \subset U$. Hence, $H$ is $G$-compact on $\bar{\Omega}$.

(3) Construct an open set $\Omega$. 
For the sake of using Lemma 2, we need to look for an appropriate open-bounded subset $\Omega \subset U$. First, we prove that the periodic solution of equation $G u=\lambda H u$ is bounded.
Corresponding to the operator equation $G u=\lambda H u$ for $\lambda \in(0,1)$, we have

$$
\left\{\begin{array}{l}
w^{\prime}(t)=\lambda\left(r(t)-A(t) e^{w\left(t-\tau_{1}(t)\right)}-\eta(t) w^{\prime}\left(t-\tau_{1}(t)\right) e^{w\left(t-\tau_{1}(t)\right)}-\frac{E(t) e^{v(t)}}{N(t) e^{v(t)}+\theta_{1}(t) e^{w(t)}}\right), \\
v^{\prime}(t)=\lambda\left(a(t)-\frac{B(t) e^{v\left(t-\tau_{2}(t)\right)}}{\theta_{2}(t) e^{w\left(t-\tau_{2}(t)\right)}}\right) .
\end{array}\right.
$$

Suppose that $u=(w(t), v(t))^{T} \in U$ is a solution of (21). Now we prove that $\|u\| \leq S^{*}, S^{*}$ is a constant.

Step 1. Prove that $|w(t)| \leq S_{1}$ and $\left|w^{\prime}(t)\right| \leq S_{2}$.

Integrating (21) over the interval $[0, T]$, we obtain

$$
\begin{aligned}
\bar{r} T= & \int_{0}^{T}\left(A(t) e^{w\left(t-\tau_{1}(t)\right)}+\eta(t) w^{\prime}\left(t-\tau_{1}(t)\right) e^{w\left(t-\tau_{1}(t)\right)}\right. \\
& \left.+\frac{E(t) e^{v(t)}}{N(t) e^{v(t)}+\theta_{1}(t) e^{w(t)}}\right) \mathrm{d} t
\end{aligned}
$$

and

$$
\bar{a} T=\int_{0}^{T}\left(\frac{B(t) e^{v\left(t-\tau_{2}(t)\right)}}{\theta_{2}(t) e^{w\left(t-\tau_{2}(t)\right)}}\right) \mathrm{d} t .
$$

In consideration of $\left[H_{1}\right]$ and $\left[H_{2}\right]$, we obtain

$$
\begin{aligned}
\int_{0}^{T} \eta(t) w^{\prime}\left(t-\tau_{1}(t)\right) e^{w\left(t-\tau_{1}(t)\right)} \mathrm{d} t & =\int_{0}^{T} \frac{\eta(t)}{1-\tau_{1}^{\prime}(t)}\left(e^{w\left(t-\tau_{1}(t)\right)}\right) \mathrm{d} t \\
& =\int_{0}^{T} \frac{\eta(t)}{1-\tau_{1}^{\prime}(t)} \mathrm{d} e^{w\left(t-\tau_{1}(t)\right)} \\
& =-\int_{0}^{T} \mu \prime(t) e^{w\left(t-\tau_{1}(t)\right)} \mathrm{d} t,
\end{aligned}
$$

which together with (22) gives

$$
\bar{r} T=\int_{0}^{T}\left((A(t)-\mu \prime(t)) e^{w\left(t-\tau_{1}(t)\right)}+\frac{E(t) e^{v(t)}}{N(t) e^{v(t)}+\theta_{1}(t) e^{w(t)}}\right) \mathrm{d} t .
$$

In view of Lemma 3 and $\left[H_{1}\right]$, we obtain

$$
\begin{aligned}
& \int_{0}^{T} A(t)-\mu \prime(t) e^{w\left(t-\tau_{1}(t)\right)} \mathrm{d} t \\
& \quad=\int_{0}^{T} \frac{\left(A\left(\beta_{1}^{-1}(t)\right)-\mu \prime\left(\beta_{1}^{-1}(t)\right)\right) e^{w(t)}}{1-\tau_{1}^{\prime}\left(\beta_{1}^{-1}(t)\right)} \mathrm{d} t
\end{aligned}
$$

$$
\begin{aligned}
\bar{r} T= & \int_{0}^{T} \frac{\left(A\left(\beta_{1}^{-1}(t)\right)-\mu \prime\left(\beta_{1}^{-1}(t)\right)\right) e^{w(t)}}{1-\tau_{1}^{\prime}\left(\beta_{1}^{-1}(t)\right)} \mathrm{d} t \\
& +\int_{0}^{T} \frac{E(t) e^{\nu(t)}}{N(t) e^{v(t)}+\theta_{1}(t) e^{w(t)}} \mathrm{d} t,
\end{aligned}
$$

which implies

$$
\bar{r} T \geq \int_{0}^{T} \frac{\left(A\left(\beta_{1}^{-1}(t)\right)-\mu \prime\left(\beta_{1}^{-1}(t)\right)\right) e^{w(t)}}{1-\tau_{1}^{\prime}\left(\beta_{1}^{-1}(t)\right)} \mathrm{d} t .
$$

Therefore,

$$
\frac{1}{T} \int_{0}^{T} e^{w(t)} \mathrm{d} t \leq \frac{\bar{r}}{\widehat{R}}
$$

where $R(t)=\left(\left(A\left(\beta_{1}^{-1}(t)\right)-\mu \prime\left(\beta_{1}^{-1}(t)\right)\right) /\left(1-\tau_{1}^{\prime}\left(\beta_{1}^{-1}(t)\right)\right)\right)$. According to (25), we obtain

$$
\bar{r} T \leq \int_{0}^{T} A(t)-\mu \prime(t) e^{w\left(t-\tau_{1}(t)\right)} \mathrm{d} t+\int_{0}^{T} \frac{E(t)}{N(t)} \mathrm{d} t,
$$

together with $\left[H_{3}\right]$, which implies

$$
\frac{1}{T} \int_{0}^{T} e^{w(t)} \mathrm{d} t \geq \frac{\bar{r}-\bar{M}}{\check{R}}
$$

where $M(t)=(E(t) / N(t))$.

According to (29) and (31), we can get that there exists $\varphi_{1} \in[0, T]$ such that

$$
\left|w\left(\varphi_{1}\right)\right| \leq \max \left\{\left|\ln \frac{\bar{r}}{\widehat{\widehat{R}}}\right|,\left|\ln \frac{\bar{r}-\bar{M}}{\check{R}}\right|\right\}:=H_{1} .
$$

By means of (25) and (29), we have

$$
\int_{0}^{T}\left[(A(t)-\mu \prime(t)) e^{w\left(t-\tau_{1}(t)\right)}+R(t) e^{w(t)}\right] \mathrm{d} t \leq 2 \bar{r} T .
$$

On the basis of the mean value theorem of integral calculus, we know that there exists $\xi \in[0, T]$, such that

$$
(A(\xi)-\mu \prime(\xi)) e^{w\left(\xi-\tau_{1}(\xi)\right)}+R(\xi) e^{w(\xi)} \leq 2 \bar{r} .
$$

Since $\bar{r}>0$, we have 


$$
\begin{aligned}
& w(\xi) \leq \ln \frac{2 \bar{r}}{\widehat{R}}, \\
& e^{w\left(\xi-\tau_{1}(\xi)\right)} \leq \frac{2 \bar{r}}{\widehat{U}}
\end{aligned}
$$

where $U=A(t)-\mu \prime(t)$.

Through the first equation of (21) and (25), we obtain

$$
\begin{gathered}
\int_{0}^{T}\left|\frac{\mathrm{d}}{\mathrm{d} t}\left(w(t)+\lambda \mu(t) e^{w\left(t-\tau_{1}(t)\right)}\right)\right| \mathrm{d} t \\
=\lambda \int_{0}^{T} \mid r(t)-\left(A(t)-\mu^{\prime}(t)\right) e^{w\left(t-\tau_{1}(t)\right)} \\
\quad-\frac{E(t) e^{v(t)}}{N(t) e^{v(t)}+\theta_{1}(t) e^{w(t)} \mid} \mathrm{d} t \\
\leq 2 \bar{r} T .
\end{gathered}
$$

Therefore,

$$
\begin{aligned}
w(t)+\lambda \mu(t) e^{w\left(t-\tau_{1}(t)\right)} \leq & w(\xi)+\lambda \mu(\xi) e^{w\left(\xi-\tau_{1}(\xi)\right)} \\
& +\int_{0}^{T}\left|\frac{\mathrm{d}}{\mathrm{d} t}\left(w(t)+\lambda \mu(t) e^{w\left(t-\tau_{1}(t)\right)}\right)\right| \mathrm{d} t \\
\leq & \ln \frac{2 \bar{r}}{\widehat{R}}+\frac{2 \bar{r} \check{\mu}}{\widehat{U}}+2 \bar{r} T:=W .
\end{aligned}
$$

So, we obtain

$$
w(t) \leq W
$$

From (36), we know

$$
\int_{0}^{T}|w(t)| \mathrm{d} t-\int_{0}^{T}\left|\frac{\mathrm{d}}{\mathrm{d} t} \lambda \mu(t) e^{w\left(t-\tau_{1}(t)\right)}\right| \mathrm{d} t \leq 2 \bar{r} T .
$$

Consequently,

$$
\begin{aligned}
\int_{0}^{T}\left|w^{\prime}(t)\right| \mathrm{d} t & \leq 2 \bar{r} T+\int_{0}^{T}\left|\left(\mu^{\prime}(t)+\eta(t) w^{\prime}\left(t-\tau_{1}(t)\right)\right) e^{w\left(t-\tau_{1}((t))\right.}\right| \mathrm{d} t \\
& \leq 2 \bar{r} T+\left|\check{w^{\prime}}\right| e^{W} T+\check{\eta} e^{W} \int_{0}^{T}\left|\mu^{\prime}(t)\right| \mathrm{d} t .
\end{aligned}
$$

Through the transpose, we can obtain

$$
\int_{0}^{T}\left|w^{\prime}(t)\right| \mathrm{d} t \leq \frac{2 \bar{r} T+|\check{\mu}| e^{W} T}{1-\check{\eta} e^{W}}:=K .
$$

Together with (32), we obtain

$$
|w(t)| \leq\left|w\left(\varphi_{1}\right)\right|+\int_{0}^{T}\left|w^{\prime}(t)\right| \mathrm{d} t \leq H_{1}+K:=S_{1} .
$$

Through the first equation of (21) and (38), we obtain

$$
\begin{aligned}
\left|w^{\prime}(t)\right|= & \mid \lambda\left(r(t)-A(t) e^{w\left(t-\tau_{1}(t)\right)}-\eta(t) w^{\prime}(t) e^{w\left(t-\tau_{1}(t)\right)}\right. \\
& \left.-\frac{E(t) e^{v(t)}}{N(t) e^{v(t)}+\theta_{1} e^{w(t)}}\right) \mid
\end{aligned}
$$

so we obtain

$$
\max \left|w^{\prime}(t)\right| \leq \check{r}+\check{A} e^{W}+\check{\eta} e^{W} \max \left|w^{\prime}(t)\right|+\frac{\check{E}}{\widehat{N}} .
$$

Utilizing $\left[\mathrm{H}_{3}\right]$, we have

$$
\left|w^{\prime}(t)\right| \leq \frac{1}{1-\check{\eta} e^{W}}\left(\check{r}+\check{A} e^{W}+\frac{\check{E}}{\widehat{N}}\right):=S_{2} .
$$

Step 2. Prove that $|v(t)| \leq S_{3}$ and $\left|v^{\prime}(t)\right| \leq S_{4}$.

By means of (23) and (38), we obtain

$$
\begin{aligned}
\bar{a} T & =\int_{0}^{T} \frac{B(t) e^{v\left(t-\tau_{2}(t)\right)}}{\theta_{2}(t) e^{w\left(t-\tau_{2}(t)\right)}} \mathrm{d} t \\
& \geq \frac{\widehat{B}}{\check{\theta}_{2}} \int_{0}^{T} \frac{e^{v(t)}}{\left(1-\tau_{2}^{\prime}\left(\beta_{2}^{-1}(t)\right)\right) e^{w(t)}} \mathrm{d} t \geq \frac{\widehat{B}}{\check{\theta}_{2} \check{\sigma} e^{W}} \int_{0}^{T} e^{v(t)} \mathrm{d} t,
\end{aligned}
$$

where $\beta_{2}(t)=t-\tau_{2}(t)$ and $\sigma(t)=1-\tau_{2}^{\prime}\left(\beta_{2}^{-1}(t)\right)$, which implies that

$$
\frac{1}{T} \int_{0}^{T} e^{v(t)} \mathrm{d} t \leq \frac{\bar{a}_{2} \check{\sigma} e^{W}}{\widehat{B}} .
$$

According to (23) and (42), we obtain

$$
\begin{aligned}
\bar{a} T & =\int_{0}^{T} \frac{B(t) e^{v\left(t-\tau_{2}(t)\right)}}{\theta_{2} e^{w\left(t-\tau_{2}(t)\right)} \mathrm{d} t} \\
& \leq \frac{\check{B}}{\widehat{\theta}_{2}} \int_{0}^{T} \frac{e^{v(t)}}{\left(1-\tau_{2}^{\prime}\left(\beta_{2}^{-1}(t)\right)\right) e^{w(t)}} \mathrm{d} t \\
& \leq \frac{\check{B}}{\widehat{\theta}_{2} \widehat{\sigma} e^{-S_{1}}} \int_{0}^{T} e^{v(t)} \mathrm{d} t .
\end{aligned}
$$

Thus,

$$
\frac{1}{T} \int_{0}^{T} e^{v(t)} \mathrm{d} t \geq \frac{\bar{a} \widehat{\theta}_{2} \widehat{\sigma} e^{-S_{1}}}{\check{B}} .
$$

From (47) and (49), there exists $\varphi_{2} \in[0, T]$ such that

$\left|v\left(\varphi_{2}\right)\right| \leq \max \left\{\left|\ln \frac{\bar{a} \check{\theta}_{2} \check{\sigma} e^{W}}{\widehat{B}}\right|,\left|\ln \frac{\bar{a} \widehat{\theta}_{2} \widehat{\sigma} e^{-S_{1}}}{\check{B}}\right|\right\}:=H_{2}$.

From (21) and (23), it is easy to see that

$$
\int_{0}^{T}\left|v^{\prime}(t)\right| \mathrm{d} t \leq 2 \bar{a} T \text {. }
$$

Then, 


$$
|v(t)| \leq\left|v\left(\varphi_{2}\right)\right|+\int_{0}^{T} v^{\prime}(t) \mathrm{d} t \leq H_{2}+2 \bar{a} T:=S_{3} .
$$

Through the second equation of (21), (42), and (52), we have

$$
\begin{aligned}
\left|v^{\prime}(t)\right| & =\left|\lambda\left(a(t)-\frac{B(t) e^{v\left(t-\tau_{2}(t)\right)}}{\theta_{2} e^{w\left(t-\tau_{2}(t)\right)}}\right)\right| \\
& \leq \check{a}+\frac{\check{B} e^{S_{3}}}{\widehat{\theta}_{2} e^{-S_{1}}}:=S_{4} .
\end{aligned}
$$

From (42), (45), (52), and (53), we obtain

$$
\|u\|=|u|_{\infty}+\left|u^{\prime}\right|_{\infty} \leq S_{1}+S_{2}+S_{3}+S_{4}:=S^{*} .
$$

At this point, we have proved that the periodic solution of equation $G u=\lambda H u$ for $\lambda \in(0,1)$ is bounded.

In the following part, we will prove that the constant solution of equation $\mathrm{QHu}=0$ is bounded. According to condition $\left[\mathrm{H}_{4}\right]$, the algebraic equation set

$$
\left\{\begin{array}{l}
\bar{r}-\bar{A} x-\frac{1}{T} \int_{0}^{T} \frac{E(t) y}{N(t) y+\theta_{1}(t) x} \mathrm{~d} t=0, \\
\bar{a}-\overline{\left(\frac{B}{\theta_{2}}\right)} \frac{y}{x}=0
\end{array}\right.
$$

has finite positive solutions $\left(x_{i}^{*}, y_{i}^{*}\right)(i=1,2, \ldots k)$. Therefore, for $u \in \operatorname{KerG}$, the operator equation $Q H u=0$ has finite positive solutions $u_{i}^{*}=\left(w_{i}^{*}, v_{i}^{*}\right)(i=1,2, \ldots k)$. Suppose that $S_{0}=\max _{1<i<k}\left\{\left|w_{i}^{*}\right|+\left|v_{i}^{*}\right|\right\}$, then $\left\|u_{i}^{*}\right\| \leq S_{0}(i=$ $1,2, \ldots k)$. Therefore, the solution of equation $\mathrm{QHu}=0$ for $u \in \operatorname{Ker} G$ is bounded.

Let $S=S^{*}+S_{0}$, and take $\Omega=\left\{u=(w, v)^{T}:\|u\|<S\right\}$. It is easy to see that $\Omega$ verifies condition (i) in Lemma 2. When $u \in \partial \Omega \cap \operatorname{Ker} G=\partial \Omega \cap R^{2}, u$ is a constant vector in $R^{2}$ with $\|u\|=S$, we obtain

$$
\mathrm{QHu}(t)=\left(\begin{array}{c}
\bar{r}-\bar{A} x-\frac{1}{T} \int_{0}^{T} \frac{E(t) y}{N(t) y+\theta_{1}(t) x} \mathrm{~d} t \\
\bar{a}-\overline{\left(\frac{B}{\theta_{2}}\right)} \frac{y}{x}
\end{array}\right) \neq 0 .
$$

Thus, condition (ii) of Lemma 2 holds.

(4) Calculate the degree of coincidence.

For each $u=(w, v)^{T} \in \operatorname{Im} Q$, define isomorphism $J: \operatorname{ImQ} \longrightarrow \operatorname{ker} G$ so that $J(u)=u$. By simply computing, we get the following Jacobian matrix:

$$
\bar{J}(w, v)=\left(\begin{array}{cc}
-\bar{A} e^{w}+\frac{1}{T} \int_{0}^{T} \frac{\theta_{1}(t) E(t) e^{v+w}}{\left(N(t) e^{v}+\theta_{1}(t) e^{w}\right)^{2}} \mathrm{~d} t & \frac{1}{T} \int_{0}^{T} \frac{-E(t) \theta_{1}(t) e^{w+v}}{\left(N(t) e^{v}+\theta_{1}(t) e^{w}\right)^{2}} \mathrm{~d} t \\
\overline{\left(\frac{B}{\theta_{2}}\right) \frac{e^{v}}{e^{w}}} & -\overline{\left(\frac{B}{\theta_{2}}\right)} \frac{e^{v}}{e^{w}}
\end{array}\right) .
$$

Then, we have

$$
\begin{aligned}
& \operatorname{det} \bar{J}(w, v)=\left(\bar{A} e^{w}-\frac{1}{T} \int_{0}^{T} \frac{\theta_{1}(t) E(t) e^{v+w}}{\left(N(t) e^{v}+\theta_{1}(t) e^{w}\right)^{2}} \mathrm{~d} t\right)\left(\frac{\bar{B}}{\theta_{2}}\right) \frac{e^{v}}{e^{w}} \\
& +\left(\frac{\bar{B}}{\theta_{2}}\right) \frac{e^{v}}{e^{w}} \cdot \frac{1}{T} \int_{0}^{T} \frac{E(t) \theta_{1}(t) e^{w+v}}{\left(N(t) e^{v}+\theta_{1}(t) e^{w}\right)^{2}} \mathrm{~d} t \\
& =\left(\frac{\bar{B}}{\theta_{2}}\right) \bar{A} e^{v}>0, \quad \forall(w, v) \in R^{2},
\end{aligned}
$$

which implies

$$
\operatorname{deg}\{J Q H, \Omega \cap \operatorname{KerG}, 0\}=\sum_{\left(w_{i}^{*}, v_{i}^{*}\right) \in Q H^{-1}(0)} \operatorname{sgn} \operatorname{det} \bar{J}, \quad\left(w_{i}^{*}, v_{i}^{*}\right)>0 .
$$

By now, we have verified that $\Omega$ satisfies all the requirements in Lemma 2. Thus, system (16) has at least one $T$-periodic solution, which implies that system (6) has at least one positive $T$-periodic solution. This completes the proof.

Remark 1. If the delay $\tau_{1}(t)$ and $\tau_{2}(t)$ both are positive constants, that is to say, condition $\left[H_{1}\right]$ holds. At this time, the delay has no effect on the existence of periodic solutions.

Remark 2. If the neutral coefficient $\rho(t)=0$, that is, $\eta(t)=0$, at this time, condition $\left[\mathrm{H}_{2}\right]$ holds. When $\left[H_{1}\right],\left[H_{3}\right]$, and $\left[H_{4}\right]$ hold, then the system has at least one positive periodic solution.

Next, we will study the predator-prey model with random disturbances.

\section{Persistence in Mean and Extinction of System (8)}

In this section, we discuss the properties of stochastic system (8) with Smith growth, the sufficient conditions for persistence and extinction are obtained. 
Lemma 4 (see [38]). Let $(x(t), y(t))$ be a solution of system (8) with initial value $(x(0), y(0))$, then $\lim _{t \rightarrow \infty}(x(t) / t)=$ $0, \quad \lim _{t \rightarrow \infty}(y(t) / t)=0, \lim _{t \longrightarrow \infty}\left(\int_{0}^{t} x(s) d B_{1}(s) / t\right)=0$, $\lim _{t \rightarrow \infty}\left(\int_{0}^{t} y(s) d B_{2}(s) / t\right)=0$ a.s.

Theorem 2. Let $(x(t), y(t))$ be a solution of system (8) with initial value $(x(0), y(0))$. If
(1) $r-\left(\sigma_{1}^{2} / 2\right)-(e / n)>0, a-\left(\sigma_{2}^{2} / 2\right)>0$, then system (8) is persistent in mean

(2) $r-\left(\sigma_{1}^{2} / 2\right)<0, a-\left(\sigma_{2}^{2} / 2\right)<0$, then system (8) goes to extinction almost surely

Proof

(1) From the first equation of system (8) and using Itô's formula, we obtain

$$
\begin{aligned}
d(\ln x+\rho x) & =\left(\left(\frac{1}{x}+\rho\right)\left(\frac{r x}{1+\rho x}\left(1-\frac{x}{K}\right)-\frac{e x y}{(n y+x)(1+\rho x)}\right)-\frac{\sigma_{1}^{2}}{2}\right) \mathrm{d} t+\sigma_{1}(1+\rho x) \mathrm{d} B_{1}(t) \\
& =\left(r\left(1-\frac{x}{K}\right)-\frac{e y}{n y+x}-\frac{\sigma_{1}^{2}}{2}\right) \mathrm{d} t+\sigma_{1}(1+\rho x) \mathrm{d} B_{1}(t) .
\end{aligned}
$$

Integrating from 0 to $t$ on both sides, we can obtain

$$
\begin{aligned}
\ln x(t)+\rho x(t)-\ln x(0)-\rho x(0)= & \left(r-\frac{\sigma_{1}^{2}}{2}\right) t-\frac{r}{K} \int_{0}^{t} x(s) \mathrm{d} s \\
& -\int_{0}^{t} \frac{e y(s)}{n y(s)+x(s)} \mathrm{d} s \\
& +\int_{0}^{t} \sigma_{1}(1+\rho x(s)) \mathrm{d} B_{1}(s) .
\end{aligned}
$$

Then,

$$
\begin{aligned}
\ln x(t)= & \left(r-\frac{\sigma_{1}^{2}}{2}\right) t-\int_{0}^{t} \frac{e y(s)}{n y(s)+x(s)} \mathrm{d} s-\frac{r}{K} \int_{0}^{t} x(s) \mathrm{d} s \\
& -\rho x(t)+\ln x(0)+\rho x(0)+M(t),
\end{aligned}
$$

where $M(t)=\int_{0}^{t} \sigma_{1}(1+\rho x(s)) \mathrm{d} B_{1}(s)$. Dividing $t$ on both sides, we have that

$$
\begin{aligned}
\frac{1}{t} \ln x(t) \geq & \left(r-\frac{\sigma_{1}^{2}}{2}-\frac{e}{n}\right)-\frac{r}{K t} \int_{0}^{t} x(s) \mathrm{d} s \\
& +\frac{M(t)}{t}-\frac{\rho x(t)}{t}+\frac{\ln x(0)}{t}+\frac{\rho x(0)}{t} .
\end{aligned}
$$

According to Lemma 4, we obtain

$$
\liminf _{t \rightarrow \infty} \frac{\int_{0}^{t} x(t) \mathrm{d} t}{t} \geq \frac{\left(r-\left(\sigma_{1}^{2} / 2\right)-(e / n)\right) K}{r}>0 .
$$

From the second equation of system (8) and using Itô's formula, we can obtain

$$
\mathrm{d}(\ln y)=\left(a-\frac{\sigma_{2}^{2}}{2}-\frac{a b y}{x}\right) \mathrm{d} t+\sigma_{2} \mathrm{~d} B_{2}(t)
$$

Integrating from 0 to $t$ on both sides, we obtain

$$
\ln y(t)-\ln y(0)=\left(a-\frac{\sigma_{2}^{2}}{2}\right) t-a b \int_{0}^{t} \frac{y(s)}{x(s)} \mathrm{d} s+\int_{0}^{t} \sigma_{2} \mathrm{~d} B_{2}(s) \text {. }
$$

Thus,

$$
a b \int_{0}^{t} \frac{y(s)}{x(s)} \mathrm{d} s=-\ln y(t)+\ln y(0)+\left(a-\frac{\sigma_{2}^{2}}{2}\right) t+\int_{0}^{t} \sigma_{2} \mathrm{~d} B_{2}(s) .
$$

Then, we can obtain

$$
\liminf _{t \rightarrow \infty} \frac{1}{t} \int_{0}^{t} \frac{y(s)}{x(s)} \mathrm{d} s \geq \frac{a-\left(\sigma_{2}^{2} / 2\right)}{a b}>0 .
$$

Because $x$ is persistent, $\liminf _{t \rightarrow \infty}(1 / t) \int_{0}^{t} y(s) \mathrm{d} s>0$. We complete the proof of (1).

(2) By (61) and (66), we can obtain

$$
\begin{aligned}
\ln x(t)+\rho x(t)-\ln x(0)-\rho x(0) & =\left(r-\frac{\sigma_{1}^{2}}{2}\right) t-\frac{r}{K} \int_{0}^{t} x(s) \mathrm{d} s-\int_{0}^{t} \frac{e y(s)}{n y(s)+x(s)} \mathrm{d} s+\int_{0}^{t} \sigma_{1}(1+\rho x(s)) \mathrm{d} B_{1}(s) \\
& \leq\left(r-\frac{\sigma_{1}^{2}}{2}\right) t+M(t), \\
\ln y(t)-\ln y(0) & =\left(a-\frac{\sigma_{2}^{2}}{2}\right) t-a b \int_{0}^{t} \frac{y(s)}{x(s)} \mathrm{d} s+\int_{0}^{t} \sigma_{2} \mathrm{~d} B_{2}(s) \\
& \leq\left(a-\frac{\sigma_{2}^{2}}{2}\right) t+\int_{0}^{t} \sigma_{2} \mathrm{~d} B_{2}(s) .
\end{aligned}
$$


Therefore,

$$
\begin{aligned}
& \limsup _{t \rightarrow \infty} \frac{\ln x(t)}{t} \leq r-\frac{\sigma_{1}^{2}}{2}<0, \\
& \limsup _{t \rightarrow \infty} \frac{\ln y(t)}{t} \leq a-\frac{\sigma_{2}^{2}}{2}<0 .
\end{aligned}
$$

So, system (8) goes to extinction almost surely.

Theorem 2 shows that if the intensity of environmental noise is small, the prey and predator will survive for a long time, and vice versa, and they will become extinct. Therefore, environmental noise is not conducive to the survival of the population.

\section{Conclusion and Numerical Simulation}

This paper mainly solves the following problems:

(1) For system (6) with neutral time delay and impulse control, first we transform it into nonpulse equivalent system by transformation: $x(t)=\prod_{0<t_{k}<t}(1-$ $\left.\theta_{1 k}\right) D_{1}(t)$ and $y(t)=\prod_{0<t_{k}<t}\left(1-\theta_{2 k}\right) D_{2}(t)$. Then, by using the coincidence degree theory, the sufficient conditions $\left[\mathrm{H}_{1}\right]-\left[\mathrm{H}_{4}\right]$ for the existence of periodic solution of the system is obtained.

(2) For the stochastic perturbation system (8) with Smith growth, the persistence and extinction of the system are discussed.

In the following part, we introduce numerical simulations to illustrate our main theoretical results. Firstly, we verify the existence of positive periodic solution of the neutral delay Leslie predator-prey model (16).

\section{Example 1}

(1) In system (16), we choose

$$
\begin{aligned}
& r(t)=4+\sin (10 \pi t), \\
& A(t)=0.4-\cos (10 \pi t), \\
& E(t)=0.2-0.5 \cos (10 \pi t), \\
& a(t)=3+\sin (10 \pi t), \\
& B(t)=0.5+\cos (10 \pi t), \\
& \eta(t)=0.1+0.01 \sin (10 \pi t), \\
& N(t)=4+\sin (10 \pi t), \\
& \theta_{1}(t)=0.8+\sin (10 \pi t), \\
& \theta_{2}(t)=0.5+\sin (10 \pi t), \\
& \tau_{1}(t)=0.2+0.01 \cos (10 \pi t), \\
& \tau_{2}(t)=0.2+0.01 \sin (10 \pi t) .
\end{aligned}
$$

By simple calculation, we know that conditions $\left[H_{1}\right]$ and $\left[H_{2}\right]$ hold, and $\check{\eta} e^{W} \approx 0.0987<1, \bar{r}=4>\bar{M} \approx$ 0.175 . Obviously $\left[H_{3}\right]$ holds. Moreover, the algebraic equations in $\left[\mathrm{H}_{4}\right]$ have only one positive solution, which together with Theorem 1 yields that Example 1 has at least one periodic solution (see Figure 1).

(2) We choose $\tau_{1}(t)=0.3$ and $\tau_{2}(t)=0.3$ in system (16), and other parameters are the same as those in Figure 1. In this case, condition $\left[H_{1}\right]$ is true because $\tau_{1}(t)$ and $\tau_{2}(t)$ are constants. Thus, there is at least one periodic solution as long as condition $\left[\mathrm{H}_{2}-\mathrm{H}_{4}\right]$ are true (see Figure 2). This shows that the existence of periodic solutions is not affected by the constant delays.

(3) We choose $\eta(t)=0$ in system (16), and other parameters are the same as those in Figure 1.

Because the neutral coefficient $\rho(t)=0$, condition $\left[\mathrm{H}_{2}\right]$ must be true. The system has at least one periodic solution as long as other conditions are true. In this case, the prey population grows in logistic mode, which indicates that there is at least one periodic solution in general impulsive system (see Figure 3).

Example 2. Next we conduct simulations about the persistence and extinction of the stochastic Smith growth model (8). Obviously, when $r>(e / n+b), a+((a \rho K / r)+1)(r-(e /(n+$ $b)))>\left(b e /(n+b)^{2}\right)$, the deterministic model corresponding to model (8) has a unique locally asymptotically stable positive equilibrium $P^{*}=\left(x^{*}, y^{*}\right)=((K / r)(r-(e /(n+b))),(K /$ $r b)(r-(e /(n+b))))$. In system $(8)$, we choose

$$
\begin{aligned}
r & =0.4, \\
a & =0.2, \\
e & =0.4, \\
n & =2, \\
\rho & =0.1, \\
b & =0.5, \\
K & =0.8 .
\end{aligned}
$$

(1) Let $\sigma_{1}=0.1, \sigma_{2}=0.1$, then we can know that $r>(e /$ $(n+b)), a+((a \rho K / r)+1) \quad(r-(e /(n+b)))>(b e /$ $\left.(n+b)^{2}\right), r-\left(\sigma_{1}^{2} / 2\right)-(e / n)>0, a-\left(\sigma_{2}^{2} / 2\right)>0$. Both stochastic system and deterministic system exist for a long time. Thus, conclusion (1) of Theorem 2 holds (see Figure 4).

(2) Let $\sigma_{1}=0.9$ and $\sigma_{2}=0.8$. By simple calculation, we can know that $r>(e /(n+b)), \quad a+((a \rho K / r)+$ 1) $(r-(e /(n+b)))>\left(b e /(n+b)^{2}\right)$, and then the deterministic system is persistent. However, $r-\left(\sigma_{1}^{2} / 2\right)<0, a-\left(\sigma_{2}^{2} / 2\right)<0$, and then the random system is extinct. Thus, conclusion (2) of Theorem 2 holds. This shows that environmental noise is harmful to the long-term survival of the population (see Figure 5). 


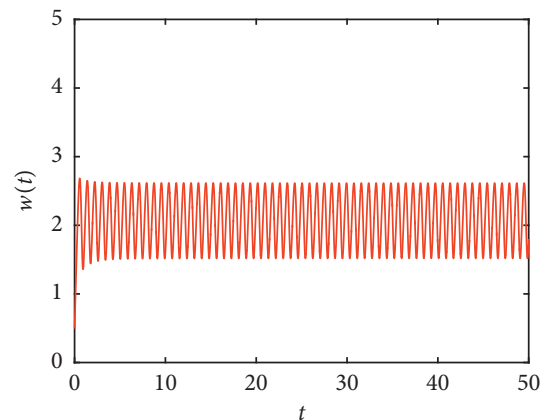

(a)

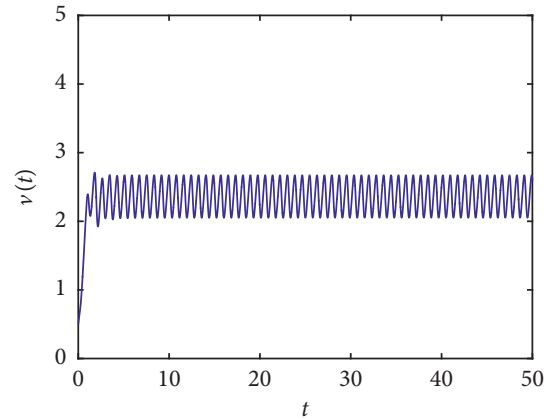

(b)

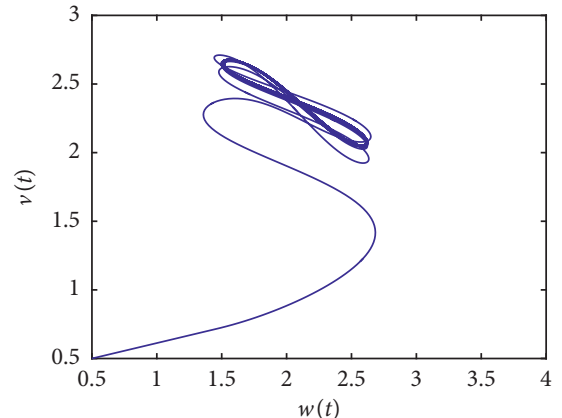

(c)

Figure 1: Computer simulation of the paths $w(t)$ and $v(t)$ for system $(16)$ with the initial value $(w(0), v(0))=(0.5,0.5)$.

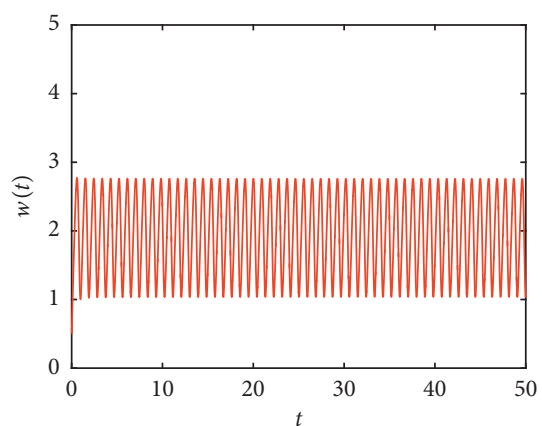

(a)

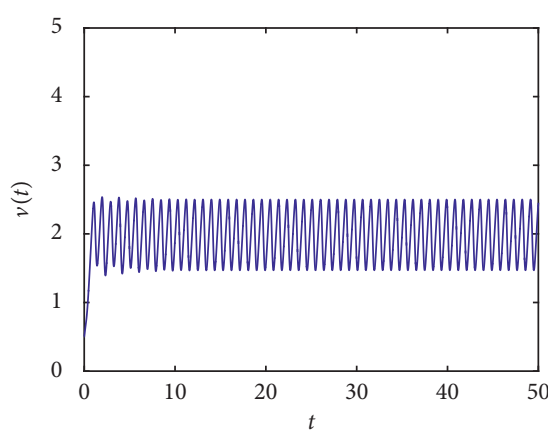

(b)

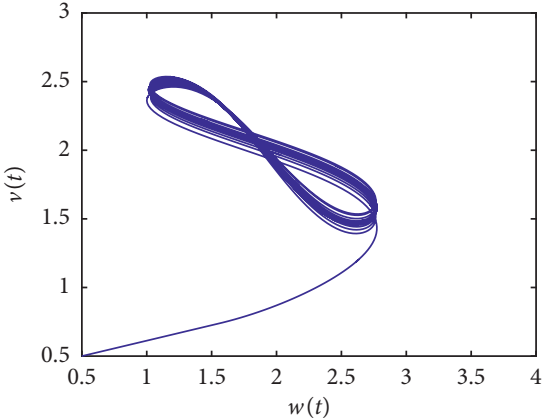

(c)

Figure 2: Computer simulation of the paths $w(t)$ and $v(t)$ for system (16) with the initial value $(w(0), v(0))=(0.5,0.5)$ and $\tau_{1}(t)=\tau_{2}(t)=0.3$

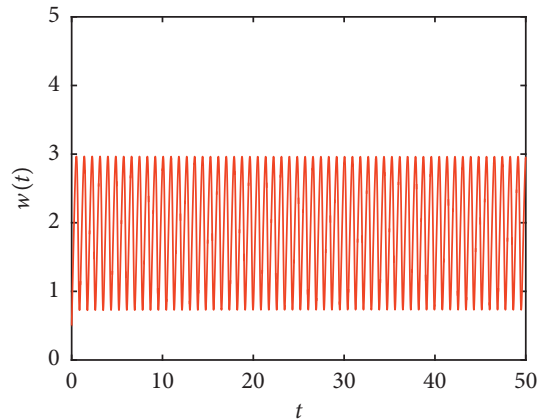

(a)

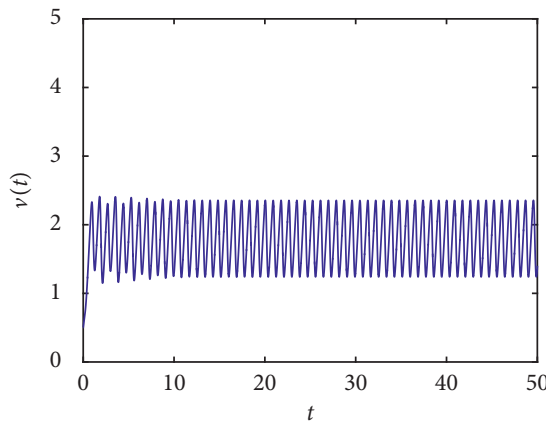

(b)

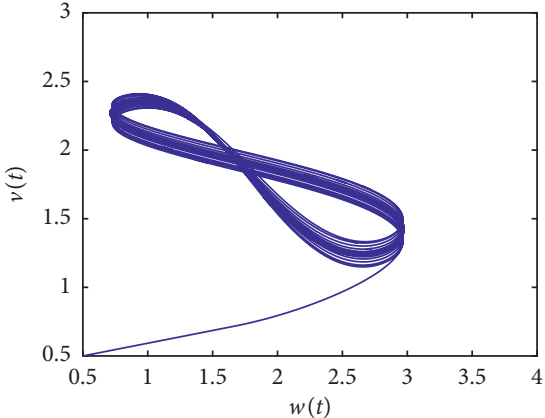

(c)

Figure 3: Computer simulation of the paths $w(t)$ and $v(t)$ for system $(16)$ with the initial value $(w(0), v(0))=(0.5,0.5)$ and $\eta(t)=0$. 


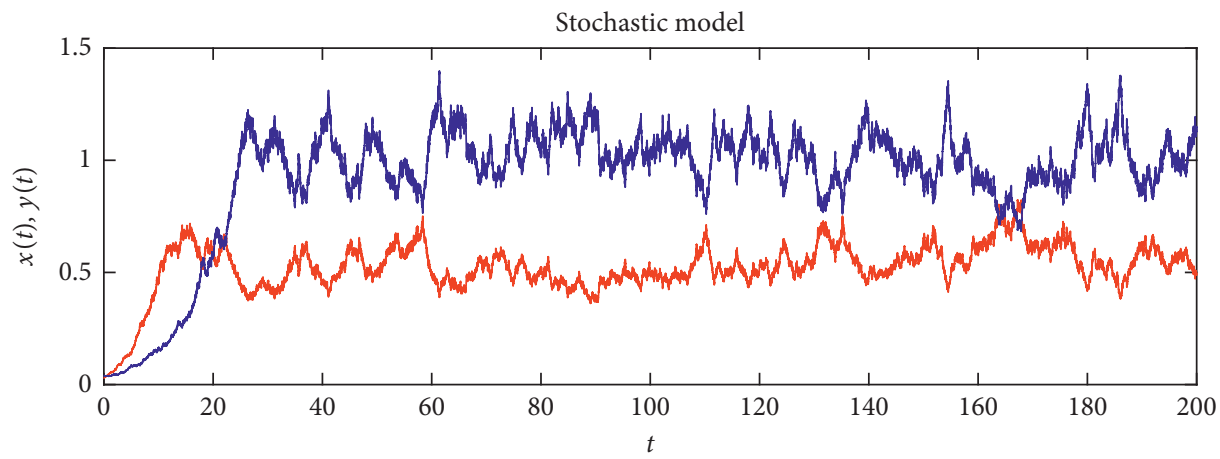

- X

(a)

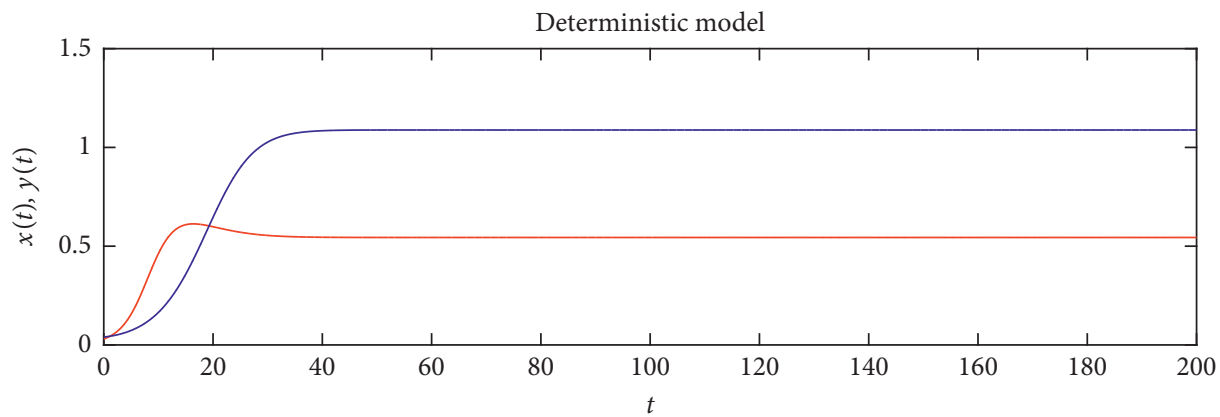

$X$
$-Y$

(b)

FIGURE 4: Computer simulations of the paths $x(t)$ and $y(t)$ for system (8) with the initial value $(x(0), y(0))=(0.03,0.04)$ and $\sigma_{1}=0.1$, $\sigma_{2}=0.1$, and its corresponding deterministic model with initial value $(x(0), y(0))=(0.03,0.04)$.

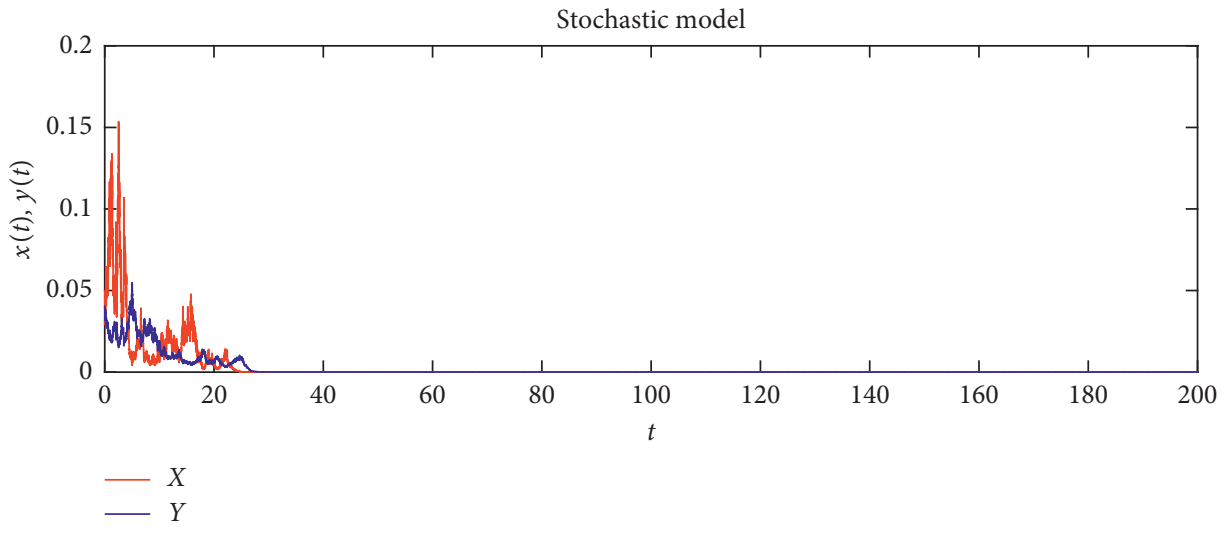

(a)

Figure 5: Continued. 


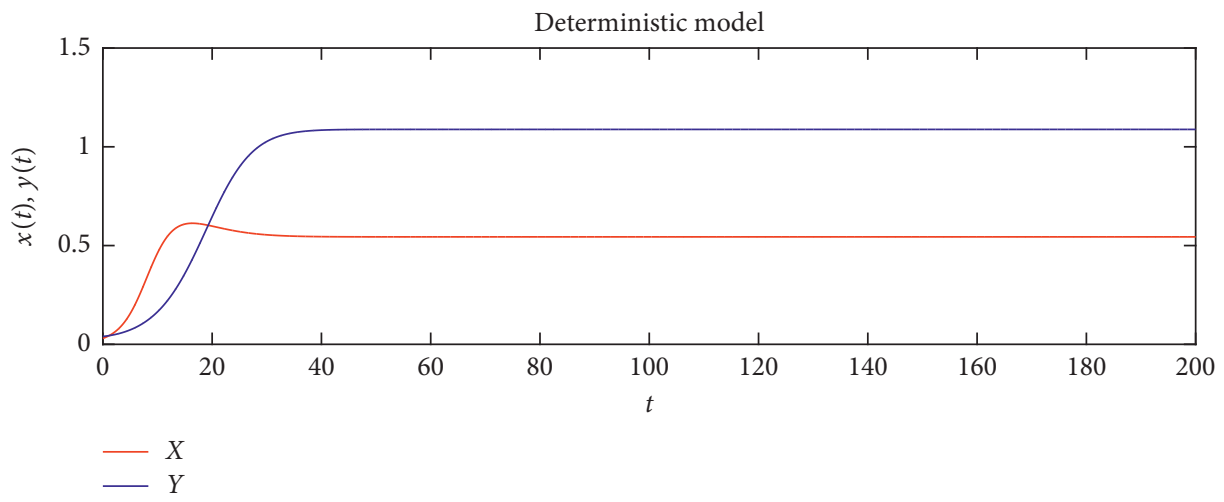

(b)

FIgURe 5: Computer simulations of the paths $x(t)$ and $y(t)$ for system (8) with the initial value $(x(0), y(0))=(0.03,0.04)$ and $\sigma_{1}=0.9$, $\sigma_{2}=0.8$, and its corresponding deterministic model with initial value $(x(0), y(0))=(0.03,0.04)$.

\section{Data Availability}

Data sharing is not applicable to this article as all datasets are hypothetical during the current study.

\section{Conflicts of Interest}

The authors declare no conflicts of interest.

\section{Authors' Contributions}

All authors contributed equally in writing this paper. All authors read and approved the final manuscript.

\section{Acknowledgments}

This work was supported by the National Natural Science Foundation of China (no. 11371230) and Shandong Provincial Natural Science Foundation of China (no. ZR2019MA003).

\section{References}

[1] R. Pearl and L. J. Reed, "On the rate of growth of the population of the united states since 1790 and its mathematical representation," Proceedings of the National Academy of Sciences, vol. 6, no. 6, pp. 275-288, 1920.

[2] F. E. Smith, "Population dynamics in Daphnia magna and a new model for population growth," Ecology, vol. 44, no. 4, pp. 651-663, 1963.

[3] Y. Kuang, "On neutral delay logistic Gause-type predatorprey systems," Dynamics and Stability of Systems, vol. 6, no. 2, pp. 173-189, 1991.

[4] P. H. Leslie, "Some further notes on the use of matrices in population mathematics," Biometrika, vol. 35 , no. 3-4, pp. 213-245, 1948.

[5] P. H. Leslie and J. C. Gower, "The properties of a stochastic model for the predator-prey type of interaction between two species," Biometrika, vol. 47, no. 3/4, pp. 219-234, 1960.

[6] C.-q. Xu, S.-l. Yuan, and T.-h. Zhang, "Confidence domain in the stochastic competition chemostat model with feedback control," Applied Mathematics-A Journal of Chinese Universities, vol. 33, no. 4, pp. 379-389, 2018.
[7] T. Zhang, T. Zhang, and X. Meng, "Stability analysis of a chemostat model with maintenance energy," Applied Mathematics Letters, vol. 68, pp. 1-7, 2017.

[8] K. Liu, T. Zhang, and L. Chen, "State-dependent pulse vaccination and therapeutic strategy in an SI epidemic model with nonlinear incidence rate," Computational and Mathematical Methods in Medicine, vol. 2019, Article ID 3859815, 10 pages, 2019.

[9] T. Ma, X. Meng, and Z. Chang, "Dynamics and optimal harvesting control for a stochastic one-predator-two-prey time delay system with jumps," Complexity, vol. 2019, Article ID 5342031, 19 pages, 2019.

[10] M. Chi and W. Zhao, "Dynamical analysis of two-microorganism and single nutrient stochastic chemostat model with monod-haldane response function," Complexity, vol. 2019, Article ID 8719067, 13 pages, 2019.

[11] D. Jia, T. Zhang, and S. Yuan, "Pattern dynamics of a diffusive toxin producing phytoplankton-zooplankton model with three-dimensional patch," International Journal of Bifurcation and Chaos, vol. 29, no. 4, Article ID 1930011, 2019.

[12] Z. Jiang, X. Bi, T. Zhang et al., "Global hopf bifurcation of a delayed phytoplankton-zooplankton system considering toxin producing effect and delay dependent coefficient," Mathematical Biosciences and Engineering, vol. 16, no. 5, pp. 3807-3829, 2019.

[13] T. Zhao and S. Tang, "Impulsive harvesting and by-catch mortality for the theta logistic model," Applied Mathematics and Computation, vol. 217, no. 22, pp. 9412-9423, 2011.

[14] B. Liu, Y. Zhang, and L. Chen, "The dynamical behaviors of a Lotka-Volterra predator-prey model concerning integrated pest management," Nonlinear Analysis: Real World Applications, vol. 6, no. 2, pp. 227-243, 2005.

[15] Y. Li, J. Cui, and X. Song, "Asymptotic behaviour of the nonautonomous competing two-species Lotka-Volterra models with impulsive effect," Journal of Biological Dynamics, vol. 3, no. 1, pp. 58-72, 2009.

[16] T. Zhang, N. Gao, T. Wang, and H. Liu, "Global dynamics of a model for treating microorganisms in sewage by periodically adding microbial flocculants," Mathematical Biosciences and Engineering, vol. 17, no. 1, pp. 179-201, 2020.

[17] Y. Li and X. Meng, "Dynamics of an impulsive stochastic nonautonomous chemostat model with two different growth rates in a polluted environment," Discrete Dynamics in Nature and Society, vol. 2019, Article ID 5498569, 15 pages, 2019. 
[18] H. Qi, X. Meng, and F. Tao, "Dynamics analysis of a stochastic non-autonomous one-predator-two-prey system with beddington-deangelis functional response and impulsive perturbations," Advances in Difference Equations, vol. 2019, no. 1, p. 235, 2019.

[19] Z. Du and Z. Feng, "Periodic solutions of a neutral impulsive predator-prey model with Beddington-DeAngelis functional response with delays," Journal of Computational and Applied Mathematics, vol. 258, pp. 87-98, 2014.

[20] X. Chen and Z. Du, "Existence of positive periodic solutions for a neutral delay predator-prey model with hassell-varley type functional response and impulse," Qualitative Theory of Dynamical Systems, vol. 17, no. 1, pp. 67-80, 2018.

[21] X. Yu and S. Yuan, "Asymptotic properties of a stochastic chemostat model with two distributed delays and nonlinear perturbation," Discrete Continuous Dynamical Systems-B, vol. 22, no. 11, 2017.

[22] M. Liu and K. Wang, "Global stability of a nonlinear stochastic predator-prey system with Beddington-DeAngelis functional response," Communications in Nonlinear Science and Numerical Simulation, vol. 16, no. 3, pp. 1114-1121, 2011.

[23] N. Gao, Y. Song, X. Wang, and J. Liu, "Dynamics of a stochastic SIS epidemic model with nonlinear incidence rates," Advances in Difference Equations, vol. 2019, no. 1, p. 41, 2019.

[24] Q. Liu, D. Jiang, T. Hayat, and B. Ahmad, "Stationary distribution and extinction of a stochastic predator-prey model with additional food and nonlinear perturbation," Applied Mathematics and Computation, vol. 320, pp. 226-239, 2018.

[25] T. Feng, Z. Qiu, X. Meng, and L. Rong, "Analysis of a stochastic HIV-1 infection model with degenerate diffusion," Applied Mathematics and Computation, vol. 348, pp. 437-455, 2019.

[26] W. Zhao, J. Liu, M. Chi, and F. Bian, "Dynamics analysis of stochastic epidemic models with standard incidence," $A d$ vances in Difference Equations, vol. 2019, no. 1, p. 22, 2019.

[27] F. Tao and Z. Qiu, "Global analysis of a stochastic TB model with vaccination and treatment," Discrete Continuous Dynamical Systems-B, vol. 24, no. 6, p. 2923, 2018.

[28] H. Ren and W. Zhao, "Dynamics analysis of a stochastic Leslie-Gower predator-prey model with feedback controls," Mathematical Problems in Engineering, vol. 2019, Article ID 8631272, 13 pages, 2019.

[29] T. Zhang, J. Wang, Y. Li, Z. Jiang, and X. Han, "Dynamics analysis of a delayed virus model with two different transmission methods and treatments," Advances in Difference Equations, vol. 2020, no. 1, pp. 1-17, 2020.

[30] G. Liu, H. Qi, Z. Chang, and X. Meng, "Asymptotic stability of a stochastic May mutualism system," Computers \& Mathematics with Applications, vol. 79, no. 3, pp. 735-745, 2020.

[31] H. Qi, X. Meng, and Z. Chang, "Markov semigroup approach to the analysis of a nonlinear stochastic plant disease model," Electronic Journal of Differential Equations, vol. 2019, no. 116, pp. 1-19, 2019.

[32] H. Zhang and T. Zhang, "The stationary distribution of a microorganism flocculation model with stochastic perturbation," Applied Mathematics Letters, vol. 103, Article ID 106217, 2020.

[33] X. Yu, S. Yuan, and T. Zhang, "Survival and ergodicity of a stochastic phytoplankton-zooplankton model with toxinproducing phytoplankton in an impulsive polluted environment," Applied Mathematics and Computation, vol. 347, pp. 249-264, 2019.

[34] X. Yu, S. Yuan, and T. Zhang, “Asymptotic properties of stochastic nutrient-plankton food chain models with nutrient recycling," Nonlinear Analysis: Hybrid Systems, vol. 34, pp. 209-225, 2019.

[35] S. Zhao, S. Yuan, and H. Wang, "Threshold behavior in a stochastic algal growth model with stoichiometric constraints and seasonal variation," Journal of Differential Equations, vol. 268, no. 9, pp. 5113-5139, 2020.

[36] R. E. Gaines and J. L. Mawhin, "Coincidence degree and nonlinear differential equations," Lecture Notes in Mathematics, Vol. 568, Springer, Berlin, Germany, 1977.

[37] S. Lu, "On the existence of positive periodic solutions to a Lotka Volterra cooperative population model with multiple delays," Nonlinear Analysis: Theory, Methods \& Applications, vol. 68, no. 6, pp. 1746-1753, 2008.

[38] X. Meng, F. Li, and S. Gao, "Global analysis and numerical simulations of a novel stochastic eco-epidemiological model with time delay," Applied Mathematics and Computation, vol. 339, pp. 701-726, 2018. 